\title{
Role of mean platelet volume and platelet/ lymphocyte ratio in assessing the severity of acute respiratory infections in children
}

\author{
Diana Madalina Chiheri', Maria Oana Sasaran², Lorena Elena Melit ${ }^{3}$ \\ 1"George Emil Palade" University of Medicine, Pharmacy, Sciences and Technology Tg. Mures, Romania \\ ${ }^{2}$ Pediatrics III, "George Emil Palade " University of Medicine, Pharmacy, Sciences and Technology, \\ Tg. Mures, Romania \\ ${ }^{3}$ Pediatrics I, "George Emil Palade" University of Medicine, Pharmacy, Sciences and Technology, \\ Tg. Mures, Romania
}

\begin{abstract}
Introduction. Acute respiratory infections are one of the most frequent medical conditions among pediatric patients. Although most evolve favorably, acute respiratory failure is one of the major complications, being the main cause responsible for increased mortality. Different and easy to access parameters, obtained as a result of a simple complete blood count, can be considered as inflammatory biomarkers, that are useful in the diagnosis and prognosis of multiple conditions, including acute respiratory infections. Mean platelet volume and the platelet/lymphocyte ratio are among these biomarkers.

Objectives. The purpose of the present study is to investigate the usefulness of the mean platelet volume and the platelet/lymphocyte ratio in distinguishing uncomplicated respiratory infections from those with potential evolution towards acute respiratory failure.

Material and methods. We conducted a prospective study on 62 patients aged between 1 month and 10 years, with various respiratory conditions, the diagnose being made based on clinical examination, laboratory findings and chest X-ray. An automated complete blood count was performed for each patient.

Results. The study group involved 26 patients with complicated respiratory infections that lead to acute respiratory failure, while the control group consisted of patients with uncomplicated acute respiratory infections $(N=36)$. Gender, rural/urban backgrounds did not differ significantly between the two groups. Mean platelet volume was significantly decreased in the study group $(p=0.0104)$, being associated with severe respiratory infections. Similar statistically significant results were obtained by comparing the same parameter in the subgroups of cases with pneumonia and acute bronchiolitis. Increased values of platelet/lymphocyte ratio were also correlated with respiratory tract infections complicated by respiratory failure $(p=0.0445)$.

Conclusions. The results of the current study indicate the usefulness of the mean platelet volume and platelet/lymphocyte ratio as predictive markers for the development of acute respiratory failure in children with respiratory pathologies. However, prospective observational studies on larger groups of patients are needed to obtain adequate, relevant results on a larger population sample.
\end{abstract}

Keywords: mean platelet volume, platelet/lymphocyte ratio, acute respiratory failure, children, inflammation

\section{INTRODUCTION}

According to the World Health Organization, respiratory infections account for $6 \%$ of the diseases in the world, and every year more than 12 million children under the age of 5 are admitted with infectious respiratory pathology, of which about 6.6 million die (1). Acute respiratory failure is one of the major complications, directly responsible for high mortality of respiratory infections in children, in the absence of rapid access to specific therapy. Oxygen therapy is the first line of treatment when oxygen saturation values are less than or equal to 92 or when the partial oxygen pressure in the blood is less than $60 \mathrm{mmHg}(2,3)$. Therefore, early recognition of potentially severe cases is necessary for risk stratification, various easily accessible parameters, obtained as a result of a simple automatic blood cell count, being useful on this matter (4). 
In any infectious process that occurs in the airways, pathogens have the ability to activate a multitude of inflammatory cytokines that mediate the immune response. A special role is played by two cytokines, tumor necrosis factor- $\alpha$ (TNF- $\alpha$ ) and interleukin-6 (IL-6) which, by stimulating the secretion of thrombopoietin, which facilitates the release of platelets into circulation. This explains the development of reactive thrombocytosis in direct association with the severity of the infection and of the inflammatory response (5). The number of lymphocytes also has an important predictive role, being decreased in the context of sepsis or bacteremia, due to apoptosis of $\mathrm{T}$ and B lymphocytes. Lymphopenia was also found to be a predictive marker of mortality $(6,7)$. Given the individual variation of these parameters, the popularization of the platelet/lymphocyte ratio as an infectious or inflammatory biomarker is understandable, its increase being correlated with various types of infectious processes, including respiratory tract infections, urinary tract infections, Helicobacter pylori infection or malaria (4). In terms of respiratory infections, the most intensively studied were the different types of pneumonia and, more recently, infection with the new SARS-Cov2 virus, with a close relationship between the platelet/lymphocyte ratio and their severity or prognosis being described $(8,9)$.

The mean platelet volume (MPV), routinely identified using automatic blood cell count, represents the size of circulating platelets, being correlated with platelet function or activation (10). MPV is influenced by the thrombopoiesis process, the size of circulating platelets being the expression of their various stages of maturation. Therefore, accelerated thrombopoiesis, which occurs in an inflammatory context, may be associated with an increase in the average value of platelet volume, MPV being extensively studied in relation to inflammatory bowel disease, ankylosing spondylitis or rheumatoid arthritis (11-13). However, there are studies that have shown a downward trend of MPV values in various pathologies, an example being acute bronchiolitis in infants or young children (14).

Through this study, we aim to establish the role of MPV and platelet/lymphocyte ratio in assessing the severity of acute respiratory infections in children. As the data on this subject are limited, the number of studies carried out on pediatric population being low, we want to determine to what extent these parameters could be used in the future to differentiate common respiratory pathologies from those with evolutionary potential towards acute respiratory failure.

\section{MATERIAL AND METHOD}

We conducted a prospective study on 62 patients with acute respiratory infections, aged between 1 month and 10 years (the number of patients hospitalized with these types of pathologies over the age of 10 years was low, being limited to a few individual cases), who were hospitalized in Pediatric Clinic I of the Tg. Mures County Emergency Clinical Hospital, between March 2019 and March 2020. The study included patients with common respiratory symptoms such as nasal obstruction, rhinorrhea, cough, dysphagia, hospitalized for moderate/severe generalized symptoms (fever, inappetence, nausea, vomiting, acute dehydration syndrome) or signs of acute respiratory distress. At least one venous blood sample was taken in each patient for automatic blood cell count, from which the mean platelet volume, absolute number of lymphocytes and platelets were recorded. Patients with known chronic conditions, including those with chronic respiratory diseases predisposed to complicated or potentially severe respiratory infections such as cystic fibrosis, bronchial asthma or pulmonary bronchodysplasia, were excluded from the study.

The division of patients into two groups, patients with uncomplicated respiratory infections and those with respiratory infections complicated by acute respiratory insufficiency was based on oxygen saturation values $(\mathrm{SaO} 2)$ of $\leq 92 \%$ in ambient air at the time of admission, subjects with these values being placed into the second category.

Statistical data analysis was done using GraphPad PrismT software. The Mann-Whitney test was used to compare averages between the two groups, as the variables studied did not comply to a Gaussian distribution. For the $p$ value, the statistical significance threshold was set at 0.05 (corresponding to a $95 \%$ confidence interval).

The study was approved by the Ethics Committee of the Tg. Mures County Emergency Clinical Hospital. Only patients whose legal guardians signed informed consent were included in the study, thus agreeing with study inclusion.

\section{RESULTS}

Out of the total study population $(\mathrm{N}=62)$, the study group comprised of patients with complicated respiratory infections with acute respiratory failure $(\mathrm{N}$ $=26$ ), while the control group included patients with uncomplicated acute respiratory infections $(\mathrm{N}=36)$. Diagnosis of respiratory conditions was established according to the clinical examination, paraclinical and radiological aspect (if chest X-ray was required). The distribution of the diagnoses of population included in 
the study is illustrated in figure 1. There is an absolute predominance of pneumonia cases, which accounted for more than half of the total population in the study (52\%), followed by cases of acute bronchiolitis (17\%).

The average age of patients included in the study was 2.44 years \pm 2.13 SD. There was no statistically significant difference between the two groups in terms of age $(p=0.3923)$. The age group distribution is depicted in fig. 2. In terms of gender distribution, the majority of patients included in the study were male, with a weight of $65 \%$, corresponding to a sex ratio of 1.88:1 (fig. 3).

The male or female sex does not influence the risk of developing acute respiratory failure, the distribu-

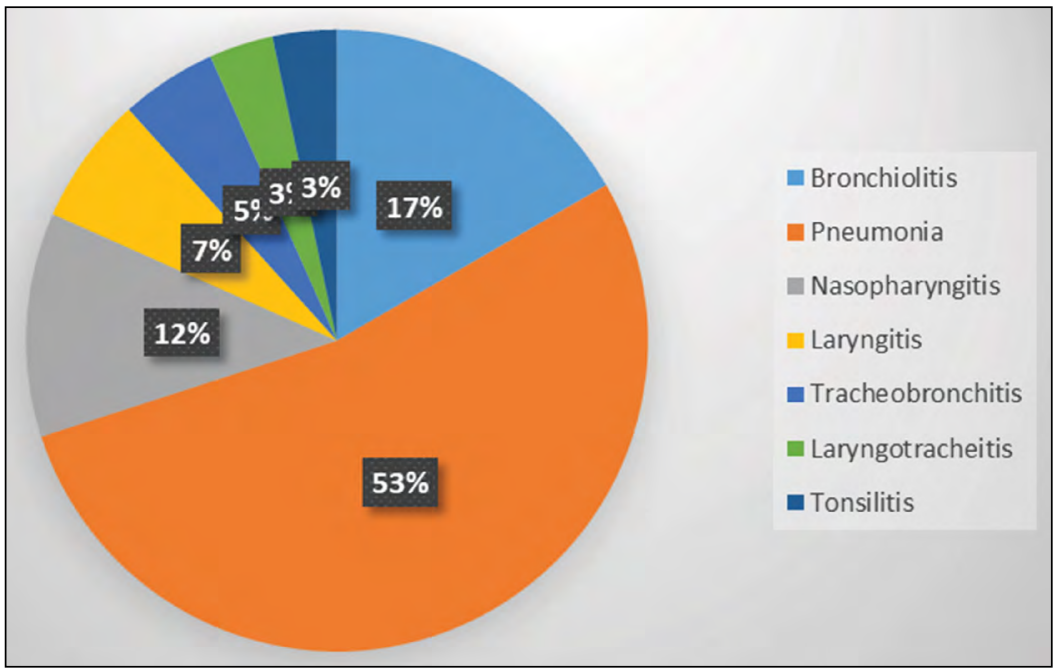

FIGURE 1. Diagnoses distribution of the entire study grp

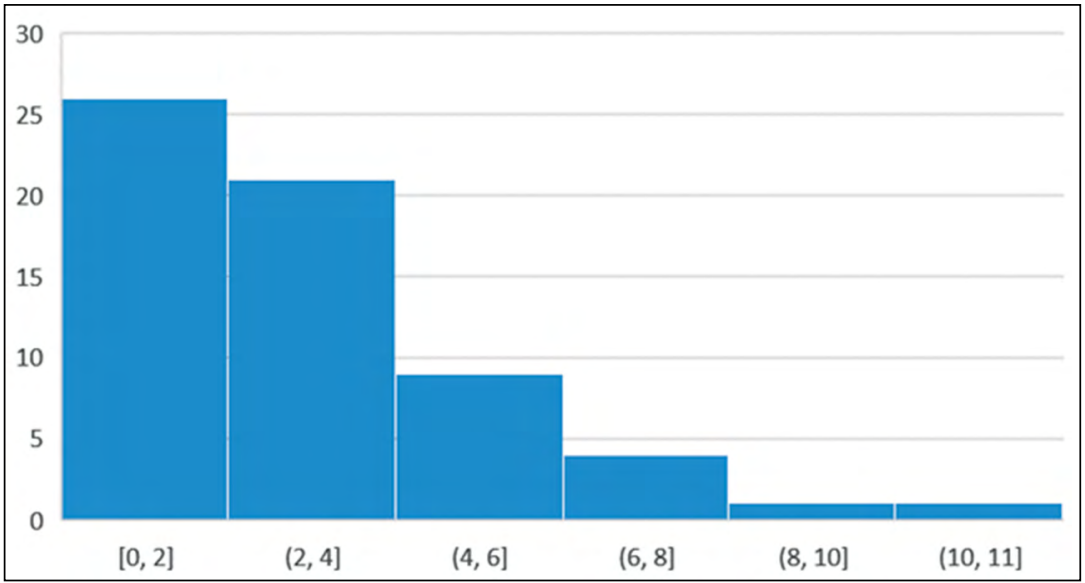

FIGURE 2. Distribution by age categories

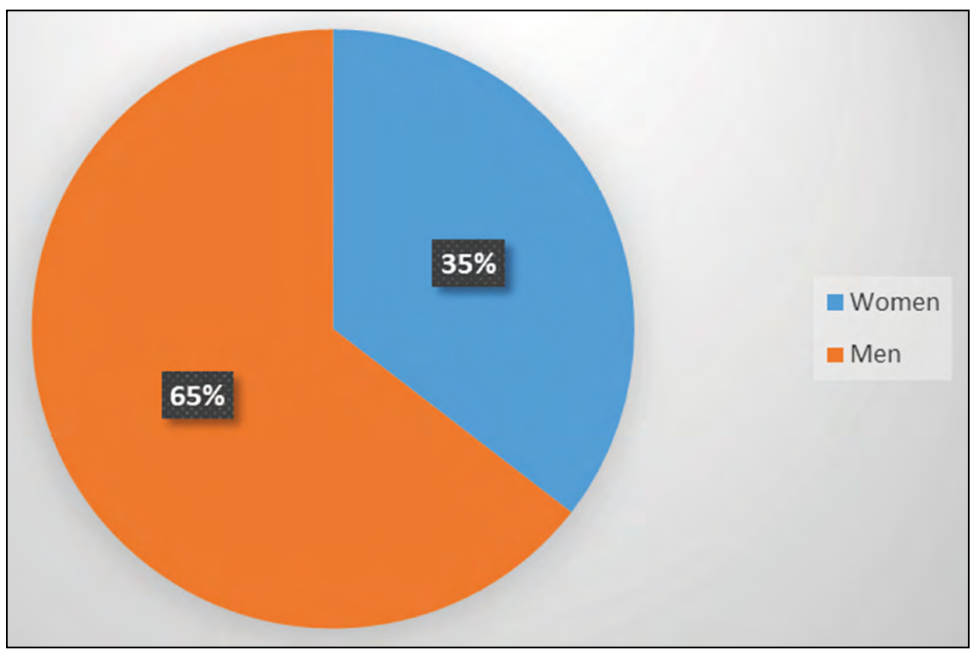

FIGURE 3. Gender distribution of the study group 
tion by sex is not significantly different between the two groups ( $\mathrm{p}=0.999, \mathrm{RR}=0.958, \mathrm{CI} 95 \%$ ) (fig. 4).

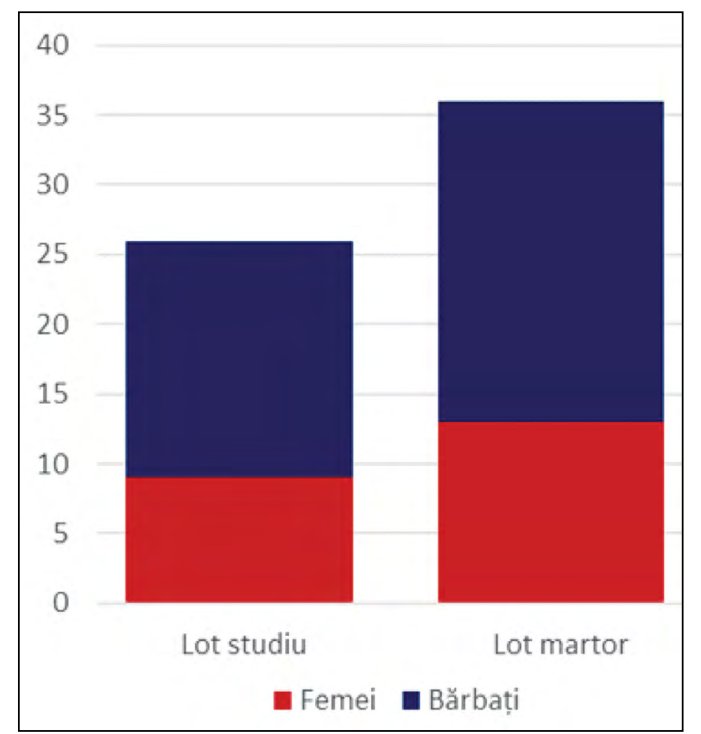

FIGURE 4. Gender distribution depending on the severity of respiratory tract infections

An identical percentage distribution to the one obtained for male/female sex of $65 \%$ and $35 \%$ was noted regarding individual background of origin (rural and urban respectively) in the study group, as seen in figure 5 .

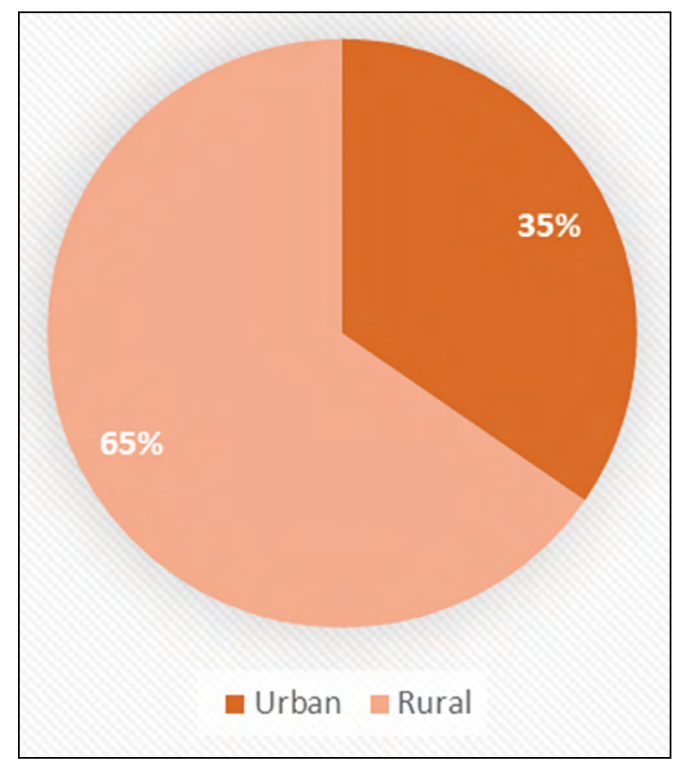

FIGURE 5. Rural/Urban background of the entire study population

However, the rural or urban background did not differ significantly between the two groups and did not influence the development of acute respiratory failure $(\mathrm{p}=0.301, \mathrm{RR}=0.692, \mathrm{CI} 95 \%)$.

Table 1 reflects the comparison of the two groups in terms of mean MPV values and platelet/lymphocyte ratio. The mean value of MPV was significantly lower in the study group (8.972 $\pm 1.868 \mathrm{SD})$ than in the control group $(9.345 \pm 1.160 \mathrm{SD}, \mathrm{p}=0.0104)$. On the other hand, the platelet/lymphocyte ratio showed significantly higher mean values in patients with acute respiratory failure than those with uncomplicated respiratory infections $(160.9 \pm 101.1 \mathrm{SD}$, compared to 113.4 $\pm 71.50 \mathrm{SD}, \mathrm{p}=0.0445)$.

TABLE 1. Comparison of parameters between the group with acute respiratory failure and the control group

\begin{tabular}{|c|c|c|l|}
\hline Parameter & $\begin{array}{c}\text { Study group } \\
\text { mean } \pm \text { SD } \\
\text { (median) }\end{array}$ & $\begin{array}{c}\text { Control group } \\
\text { mean } \pm \text { SD } \\
\text { (median) }\end{array}$ & P value \\
\hline $\begin{array}{c}\text { Mean platelet } \\
\text { volume }\end{array}$ & $\begin{array}{c}8.972 \pm 1.868 \\
(8,600)\end{array}$ & $\begin{array}{c}9.345 \pm 1.160 \\
(9,400)\end{array}$ & $\mathrm{p}=0.0104$ \\
\hline $\begin{array}{c}\text { Trombocyte/ } \\
\text { Lymphocytes } \\
\text { ratio }\end{array}$ & $\begin{array}{c}160.9 \pm 101.1 \\
(137.8)\end{array}$ & $\begin{array}{c}113.4 \pm 71.50 \\
(86.27)\end{array}$ & $\mathrm{p}=0.0445$ \\
\hline
\end{tabular}

Since the number of patients with pathologies different from pneumonia and acute bronchiolitis (with increased prevalence among the subjects included in the study) was reduced, we considered it inappropriate to analyze the two parameters for individual cases of other types of conditions. Similar results to those obtained in the analysis of the two groups were obtained for MPV values, for the subgroups of bronchiolitis and pneumonia (table 2), which were considerably lower in patients with acute respiratory failure ( $p$ $=0.0159$ and $\mathrm{p}=0.0224$, respectively).

TABLE 2. Comparison of MPV values for patients diagnosed with pneumonia or bronchiolitis

\begin{tabular}{|c|c|c|c|}
\hline $\begin{array}{c}\text { Mean platelet } \\
\text { volume }\end{array}$ & $\begin{array}{c}\text { Study group } \\
\text { mean } \pm S D \\
\text { (median) }\end{array}$ & $\begin{array}{c}\text { Control group } \\
\text { mean } \pm S D \\
\text { (median) }\end{array}$ & P value \\
\hline Pneumonia & $\begin{array}{c}8.427 \pm 1.265 \\
(8,600)\end{array}$ & $\begin{array}{c}9.151 \pm 1.358 \\
(9,300)\end{array}$ & $p=0.0224$ \\
\hline Bronchiolitis & $\begin{array}{c}8.660 \pm 0.6025 \\
(8,500)\end{array}$ & $\begin{array}{c}10.22 \pm 0.8729 \\
(10.20)\end{array}$ & $p=0.0159$ \\
\hline
\end{tabular}

The platelet/lymphocyte ratio for patients diagnosed with pneumonia did not show significantly different values in subjects with acute respiratory failure $(\mathrm{p}=0.266)$. For the subgroup of patients diagnosed with bronchiolitis, the results varied upwards, with the study group showing higher values of the platelet/ lymphocyte ratio than the control group, but lacked significant statistical correlations (table 3 ).

TABLE 3. Comparison of platelet/lymphocyte ratio values for patients diagnosed with pneumonia or bronchiolitis

\begin{tabular}{|c|c|c|c|}
\hline $\begin{array}{c}\text { Platelet/ } \\
\text { lymphocyte } \\
\text { ratio }\end{array}$ & $\begin{array}{c}\text { Study group } \\
\text { mean } \pm S D \\
\text { (median) }\end{array}$ & $\begin{array}{c}\text { Control group } \\
\text { mean } \pm S D \\
\text { (median) }\end{array}$ & P value \\
\hline Pneumonia & $156.5 \pm 88.54$ & $119.9 \pm 68.94$ & $\mathrm{p}=0.2666$ \\
& $(140.4)$ & $(105.3)$ & \\
\hline Bronchiolitis & $122.4 \pm 77.61$ & $56.70 \pm 15.16$ & $\mathrm{p}=0.3095$ \\
& $(106.9)$ & $(57.50)$ & \\
\hline
\end{tabular}




\section{DISCUSSIONS}

Hematological parameters such as MPV or platelet/lymphocyte ratio, together with neutrophil/lymphocyte ratio, monocyte/lymphocyte ratio or erythrocytic indices have become increasingly studied in relation to the diagnosis or prognosis of various pathologies. Although the majority of studies available in the literature were carried out on adult patients, platelet/lymphocyte ratio or MPV have been reported as reliable inflammatory biomarkers in various pediatric age-specific conditions, such as acute articular rheumatism (15), measles (16), acute appendicitis (17), Henoch-Schönlein purpura (18) or type 1 diabetes mellitus (19). However, the usefulness of these parameters is questioned by many authors, who dispute their relevance due to the lack of standardization of reference intervals in healthy people. A study of a large population in South Korea attempted to establish reference intervals for the above parameters, in relation to age and sex, but included predominantly adult patients and produced a single age group under 19 years for the pediatric population. The authors stressed the need to develop such studies on populations from different regions of the world, as their proposed values may vary by race and geographical area (20). Another study, conducted on a healthy male population in Iran, found that the baseline ranges of MPV vary significantly depending on age (21). Therefore, in the absence of reference values on the healthy population, differences in mean values of various hematological parameters between healthy children and those with various pathologies are difficult to analyze. Through this study, we wanted to prospectively investigate the clinical significance of MPV and platelet/ lymphocyte ratio in terms of predictability of aggravation of acute respiratory infections in children. The lack of inclusion of a healthy patient as a reference group can be motivated by the difficulty of obtaining data from subjects without pathologies in the hospital environment and by the reference intervals issue, previously exposed.

MPV has been investigated in relation to multiple respiratory conditions, one of the most intensively studied being tuberculosis. Esra Ertan Yazar et al. obtained significantly lower levels of MPV in patients with active pulmonary tuberculosis compared to healthy individuals, but concluded that this parameter is not a significant marker in assessing the severity of such an infection (22). MPV values were also studied in relation to bronchiolitis. A study in Turkey on 284 patients reported higher levels of MPV in the group of patients with bronchiolitis than in healthy patients. The authors explained this result by the stimulation of thrombopoiesis in inflammatory context, leading to the release of immature platelet precursors with high cellular volume in circulation (23). The results obtained in our study are contrary to the conclusions of the above mentioned study, as severe forms of bronchiolitis, associated with acute respiratory failure, appear to influence the values of MPV downwards. On the other hand, there are also studies that support our hypothesis, proving an association between bronchiolitis, infection with respiratory syncytial virus and decreased MPV levels $(14,24)$.

Karadag-Oncel et al. demonstrated that MPV has significantly lower mean values in patients diagnosed with pneumonia compared to the control group of healthy subjects but without analyzing the differences in values depending on the severity of respiratory manifestations (25). Similar to this study, we obtained lower mean values of MPV in our study, in the subgroup of complicated pneumonias with acute respiratory failure compared to the control group.

Regarding platelet/lymphocyte ratio, Jong Han Lee et al. concluded in a retrospective study that this parameter can be used as a predictive inflammatory marker of the severity of pneumonia, since the highest values of this ratio, with strong statistical significance, were recorded in patients requiring hospitalization in the intensive care units (26). Similarly to this study, we obtained a significant increase in the same biomarker in patients with pneumonias complicated with acute respiratory failure.

This study has its limitations, the main limitation being the small number of patients included, who are part of a single geographical area. Although the children included in the study had various types of respiratory infections, the majority of patients were diagnosed with pneumonia and bronchiolitis, with the rest of the conditions representing insignificant percentages, unworthy of individual analysis. It would therefore be necessary to expand the research on a larger pediatric population sample, from different geographical areas and with a representative number of various respiratory conditions (that have not been sufficiently investigated in our study) in order to achieve statistically relevant results. However, our study is one of the few of its kind performed in the pediatric population and the only one to date that has analyzed MPV and platelet/lymphocytes ratio in relation to the severity of respiratory diseases.

\section{CONCLUSIONS}

This study proved the usefulness of mean platelet volume and platelet/ lymphocyte ratio as predictive markers of the severity of acute respiratory infections 
in children, especially in pneumonia and acute bronchiolitis cases complicated by acute respiratory failure. However, given the limited number of patients enrolled in the study, it is necessary to expand the re-

\section{REFERENCES}

1. Tazinya AA, Halle-Ekane GE, Mbuagbaw LT et al. Risk factors for acute respiratory infections in children under five years attending the Bamenda Regional Hospital in Cameroon. BMC Pulmonary Medicine. 2018;18(1):7.

2. Piteau S. Update in pediatrics. Ontario: Springer International Publishing, 2018.

3. Nitu ME, Eigen H. Respiratory failure. Pediatrics in Review. 2009;30(12):470-8.

4. Russell CD, Parajuli A, Gale HJ, et al. The utility of peripheral blood leucocyte ratios as biomarkers in infectious diseases: A systematic review and meta-analysis. Journal of Infection. 2019;78(5):339-48.

5. Kaushansky K. The molecular mechanisms that control thrombopoiesis. Journal of Clinical Investigation. 2005; 115:3339-47.

6. le Tulzo Y, Pangault C, Gacouin A et al. Early circulating lymphocyte apoptosis in human septic shock is associated with poor outcome. Shock. 2002;18(6):487-94.

7. Wyllie DH, Bowler ICJW, Peto TEA. Relation between lymphopenia and bacteraemia in UK adults with medical emergencies. Journal of Clinical Pathology. 2004;57(9):950-5.

8. Qu R, Ling Y, Zhang Y, Hui Zhi et al. Platelet-to-lymphocyte ratio is associated with prognosis in patients with coronavirus disease-19. Journal of Medical Virology. 2020; jmv.25767.

9. Kartal O, At K. Value of neutrophil to lymphocyte and platelet to lymphocyte ratios in pneumonia. Science Edition Bratisl Med J. 2017;118(9):513-6.

10. Bath PMW, Butterworth RJ. Platelet size: Measurement, physiology and vascular disease. In: Blood Coagulation and Fibrinolysis. Lippincott Williams and Wilkins; 1996. p. 157-61.

11. Kisacik B, Tufan A, Kalyoncu U et al. Mean platelet volume (MPV) as an inflammatory marker in ankylosing spondylitis and rheumatoid arthritis. Joint Bone Spine. 2008;75(3):291-4.

12. Milovanovic M, Nilsson E, Järemo P. Relationships between platelets and inflammatory markers in rheumatoid arthritis. Clinica Chimica Acta. 2004;343(1-2):237-40.

13. Kapsoritakis AN, Koukourakis MI, Sfiridaki A et al. Mean platelet volume: A useful marker of inflammatory bowel disease activity. The American Journal of Gastroenterology. 2001;96(3):776-81.

14. Ergül AB, Torun YA, Uytun S, Aslaner H, Kısaaslan AP, Şerbetçi MC. Reduction in mean platelet volume in children with acute bronchiolitis. Turk Pediatri Arsivi. 2016;51(1):40-5. search on larger pediatric populations and to analyze the two parameters individually on a more varied range of respiratory conditions.

Conflict of interest: none declared Financial support: none declared

15. Giray D, Hallioglu O. Are there any novel markers in acute rheumatic fever: Neutrophil-to-lymphocyte ratio, platelet-to-lymphocyte ratio, and monocyte-to-lymphocyte ratio. Cardiology in the Young. 2020;

16. Solmaz A, Demir A, Gümüş H, Aksoy M, Solmaz F. Neutrophil/ Lymphocyte Ratios, Platelet/Lymphocyte Ratios, and Mean Platelet Volume Values in Patients with Measles. Cureus. 2020;12(1).

17. Bozlu G. Role of mean platelet volume-to-lymphocyte ratio in the diagnosis of childhood appendicitis. Arch Argent Pediatr. 2019;117(6).

18. Özdemir ZC, Çetin N, Kar YD et al. Hematologic Indices for Predicting Internal Organ Involvement in Henoch-Schönlein Purpura (IgA vasculitis). Journal of Pediatric Hematology/Oncology. 2020; 42(1):e46-9.

19. Baghersalimi A, Koohmanaee S, Darbandi B et al. Platelet Indices Alterations in Children with Type 1 Diabetes Mellitus. Journal of Pediatric Hematology/Oncology. 2019;41(4):e227-32.

20. Lee JS, Kim NY, Na SH et al. Reference values of neutrophillymphocyte ratio, lymphocyte-monocyte ratio, platelet-lymphocyte ratio, and mean platelet volume in healthy adults in South Korea. Medicine. 2018;97(26).

21. Ghazizadeh H, Kathryn Bohn M, Kardagh Polus R et al. Comprehensive hematological reference intervals in a healthy adult male population. Cellular and Molecular Biology. 2020;66(2):99.

22. Gunluoglu G, Yazar EE, Veske NS et al. Mean platelet volume as an inflammation marker in active pulmonary tuberculosis. Multidisciplinary Respiratory Medicine. 2014;9(1):11.

23. Gökçe Ş, Kurugöl Z, Suner A. The role of mean platelet volume in the early detection of acute bronchiolitis: A prospective study. Clinical Respiratory Journal. 2018;12(10):2513-8.

24. Renshaw AA, Drago B, Toraya N et al. Respiratory syncytial virus infection is strongly correlated with decreased mean platelet volume. International Journal of Infectious Diseases. 2013 Sep;17(9).

25. Karadag-Oncel E, Ozsurekci Y, Kara A et al. The value of mean platelet volume in the determination of community acquired pneumonia in children. Italian journal of pediatrics. 2013;39(1):16.

26. Lee JH, Song S, Yoon SY et al. Neutrophil to lymphocyte ratio and platelet to lymphocyte ratio as diagnostic markers for pneumonia severity. British Journal of Biomedical Science. 2016;73(3):140-2. 